\title{
Effects of miniemulsion operation conditions on the immobilization of BSA onto PMMA nanoparticles
}

\author{
Izabella Campos $^{1}$ (D), Thamiris Paiva ${ }^{1}$ (D), Helen Ferraz ${ }^{1}$ (D) and José Carlos Pinto ${ }^{1 *}$ (D) \\ ${ }^{1}$ Programa de Engenharia Química - COPPE, Universidade Federal do Rio de Janeiro - UFRJ, Rio de \\ Janeiro, RJ, Brasil \\ *pinto@peq.coppe.ufrj.br
}

\begin{abstract}
Polymer nanoparticles have been widely used in many biomedical applications, constituting a major incentive for immobilization of proteins. Poly(methyl methacrylate) nanoparticles were synthesized through miniemulsion polymerizations and used as supports for bovine serum albumin immobilization. Particularly, the effects of surfactant type (anionic sodium dodecyl sulfate and cationic cetyl trimethyl ammonium bromide) surfactant concentration and monomer holdup on some of the final nanoparticle properties (particle sizes, zeta potential and protein load) were characterized with help of statistical experimental designs for the first time. Results showed that the characteristics of the surfactant controlled the BSA adsorption efficiency, with enhanced rates of adsorption on the anionic particle surfaces, showing that the surfactant exerts fundamental effect on functionalization of emulsified polymer particles, which must be explicitly acknowledged in studies of polymer particle functionalization with proteins. Finally, BSA adsorption was shown to follow a multilayer process, given the better fitting with the Freundlich model.
\end{abstract}

Keywords: methyl methacrylate, miniemulsion polymerization, surfactant, protein adsorption, statistical experimental design.

How to cite: Campos, I., Paiva, T., Ferraz, H., \& Pinto, J. C. (2019). Effects of miniemulsion operation conditions on the immobilization of BSA onto PMMA nanoparticles. Polimeros: Ciência e Tecnologia, 29(3), e2019036. https:// doi.org/10.1590/0104-1428.05818

\section{Introduction}

The use of polymer nanoparticles (NPs) constitutes an interesting alternative for treatment of several diseases, particularly when it is intended to increase the absorption efficiency of encapsulated drugs and to provide protection for the bioactive principle in contact with body fluids. Furthermore, NPs can ensure the controlled release of the encapsulated drug through the polymer matrix. The possibility to develop site-specific targeted drug delivery systems through bioconjugation procedures constitutes an additional advantage of using polymer NPs in biomedical applications ${ }^{[1-3]}$.

Different techniques can be used to produce polymer NPs loaded with biomedical compounds, including miniemulsion polymerizations ${ }^{[4,5]}$. It must be emphasized that advances in the process control field make possible the production of polymer NPs with controlled properties, such as shape, size, surface charge and functionalization ${ }^{[6]}$. During the production of polymer NPs in miniemulsion processes, the addition of surfactants is mandatory to stabilize the high amount of energy stored in the interfaces ${ }^{[7]}$. It is important to observe that surfactants exert significant impact on the properties of the polymer NPs, affecting the applicability of the final product and modulating the therapeutic response in drug delivery applications ${ }^{[8]}$. Despite that, quantitative analyses of surfactant effects of the immobilization of proteins onto nanoparticle surfaces produced through miniemulsion polymerizations have not been performed yet.

The production of polymer NPs loaded with drugs through miniemulsion polymerizations has already been reported by many authors. For instance, Lorca et al. ${ }^{[9]}$ produced PMMA NPs loaded with benzophenone-3 to be used in sunscreen formulations. This technique was also used by Fonseca et al. ${ }^{[10]}$ to encapsulate the drug praziquantel (used in schistosomiasis treatments) in PMMA NPs. Moreira et al. ${ }^{[1]}$ encapsulated the drug tamoxifen (used in cancer treatment) in PMMA NPs through in situ miniemulsion polymerizations. However, in these previously published studies, detailed analyses of the surfactant effects on the final polymer NPs properties were not performed.

Based on the previous paragraphs, the main objective of the present study was to perform a quantitative investigation of protein immobilization onto PMMA NPs produced through miniemulsion polymerizations, with help of experimental design tools. To the best of our knowledge, this has never been performed in previous publications. In order to do that, two different surfactants were employed: the anionic surfactant SDS (sodium dodecyl sulfate) and the cationic surfactant CTAB (cetyl trimethyl ammonium bromide), frequently used to perform miniemulsion polymerization ${ }^{[12]}$, as it is well known that proteins are sensitive to the local surface charges during the immobilization process ${ }^{[13]}$. Initially, the influence of surfactant type and concentration on the polymer NPs properties (particle sizes and zeta 
potential) were evaluated, in order to provide benchmark information for posterior quantitative analyses related to protein immobilization.

\section{Materials and Methods}

\subsection{Materials}

Monomer methyl methacrylate (MMA, with minimum purity of $99.5 \mathrm{wt} \%$ ), surfactant SDS (with minimum purity of $99 \mathrm{wt} \%$ and water content of $10 \mathrm{wt} \%$ ), initiator potassium persulfate (KPS, with minimum purity of $99 \mathrm{wt} \%$ ), co-stabilizer hexadecane (minimum purity of $99.5 \mathrm{wt} \%$ ), $\mathrm{pH}$ buffer sodium bicarbonate (minimum purity of $99.7 \mathrm{wt} \%$ ) and inhibitor hydroquinone (minimum purity of $99 \mathrm{wt} \%$ ) were supplied by VETEC Chemistry (Rio de Janeiro, Brazil). Surfactant CTAB (minimum purity of $99 \mathrm{wt} \%$ ) was purchased from REAGEN (Colombo, Brazil). Protein BSA (minimum purity of $98 \mathrm{wt} \%$ ) was supplied by Sigma-Aldrich (Missouri, USA). All chemicals were used as received, without further purification.

\subsection{Synthesis of PMMA NPS}

PMMA NPs were produced by miniemulsion polymerization. The aqueous phase was prepared by solubilizing the surfactant (SDS or CTAB) and sodium bicarbonate $(0.1 \mathrm{wt} \%$ in respect to water) in distilled water under magnetic stirring for $10 \mathrm{~min}$. The organic phase, containing MMA and hexadecane $(0.4 \mathrm{wt} \%$ in relation to the monomer $)$, was prepared separately and added into the previously prepared aqueous phase under magnetic stirring for about $10 \mathrm{~min}$, in order to form a pre-emulsified mixture. The miniemulsion was obtained through sonication for 5 min with amplitude of $20 \%(80 \mathrm{~W})$ using a Branson Sonifier (Model 102C, Danbury, USA). To prevent the temperature increase during sonication, the vessel used for emulsification was placed inside an ice bath. The miniemulsion was then transferred to a mini-reactor (Mettler Toledo, Model EasyMaxTM 102, São Paulo, Brazil) and heated. The initiator potassium persulfate $(0.5 \mathrm{wt} \%$ in respect to the monomer) was added when the desired reaction temperature was reached, and then it was assumed that the reaction started. Reaction runs were carried out at $80{ }^{\circ} \mathrm{C}$ under stirring of $500 \mathrm{rpm}$ for 2 hours. At the end of the reaction step, samples of the reaction medium were collected for evaluation of monomer conversion by gravimetry. The polymerization was halted with the addition of $1 \mathrm{wt} \%$ aqueous hydroquinone solution to collected samples.

In order to evaluate the effects of the operation parameters on the final properties of the produced PMMA NPs, a $2^{3}$ factorial design was proposed, with replicates at the central points of the continuous variables (Table 1).

Table 1. Experimental levels of analyzed parameters.

\begin{tabular}{lcc}
\hline \multicolumn{1}{c}{ Variable } & $\begin{array}{c}\text { Minimum } \\
(\mathbf{- 1})\end{array}$ & $\begin{array}{c}\text { Maximum } \\
(+\mathbf{1})\end{array}$ \\
\hline Surfactant $\left(\mathrm{x}_{1}\right)$ & SDS & CTAB \\
Surfactant concentration $(\mathrm{wt} \%)^{\mathrm{a}}\left(\mathrm{x}_{2}\right)$ & 1 & 5 \\
Mass ratio of $\mathrm{O} / \mathrm{W}^{\mathrm{b}}$ phases $(\%)\left(\mathrm{x}_{3}\right)$ & 15 & 25 \\
\hline
\end{tabular}

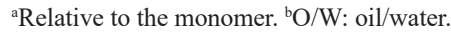

As the type of surfactant is a discrete variable and was one of the analyzed design variables $\left(\mathrm{x}_{1}\right)$, replicates were performed at the central point of the continuous variables for both discrete variables (SDS and CTAB). This type of experimental plan allows for independent evaluation of the experimental effects and proper characterization of the experimental reproducibility, assumed to be constant in the analyzed experimental grid $^{[14]}$. Particle sizes, zeta potential and BSA adsorption were used as experimental responses. The obtained experimental results were analyzed and the significance of each process parameter was determined with help of statistical models.

\subsection{Characterization}

Average particle sizes, polydispersity index (PdI) and zeta potential were measured through dynamic light scattering (DLS), using a Zetasizer Nano ZS (Malvern, Worcestershire, UK). The size distributions of both PMMA NPs and emulsified MMA droplets were analyzed. Characterization tests were performed with dilution of 1:500 (v/v) in distilled water at $25^{\circ} \mathrm{C}$. BSA adsorption tests were conducted with the produced latex, using $25 \mathrm{mg}$ of PMMA NPs per assay. $10 \mathrm{~mL}$ of $1 \mathrm{mg} \cdot \mathrm{mL}^{-1}$ BSA solution, previously prepared in acetate buffer $(\mathrm{pH}=4.5)$, were added into tubes containing the latex and kept under mild stirring for 24 hours at room temperature. This time is sufficient to achieve the maximum BSA adsorption, as previously determined through more detailed kinetic studies ${ }^{[15]}$. Afterwards, the solid phase was separated through centrifugation (ThermoScientific, Model Megafuge 16R, Waltham, USA) using an AMICON ${ }^{\circledR}$ 100K device (Millipore, Darmstadt, Germany). The BSA concentration in the filtrate was evaluated with standard Bradford assays ${ }^{[16]}$. The adsorbed BSA was quantified through mass balance and reported as the mass of BSA per surface area of the NPs, assuming the spherical geometry, as reported in previous studies ${ }^{[17]}$. The BSA adsorption isotherms were evaluated by varying the initial BSA concentration in the range from 0.1 to $5.0 \mathrm{mg} \cdot \mathrm{mL}^{-1}$. Experimental data were fitted using the standard Langmuir (Equation 1), Freundlich (Equation 2) and Langmuir-Freundlich (Equation 3) models $^{[18-20]}$ :

$$
\begin{aligned}
& \mathrm{q}_{\mathrm{e}}=\frac{\mathrm{q}_{\mathrm{mL}} \mathrm{k}_{\mathrm{L}} \mathrm{C}_{\mathrm{eq}}}{1+\mathrm{k}_{\mathrm{L}} \mathrm{C}_{\mathrm{eq}}} \\
& \mathrm{q}_{\mathrm{e}}=\mathrm{q}_{\mathrm{mF}} \mathrm{k}_{\mathrm{F}} \mathrm{C}_{\mathrm{eq}}^{1 / \mathrm{n}_{\mathrm{F}}} \\
& \mathrm{q}_{\mathrm{e}}=\frac{\mathrm{q}_{\mathrm{mLF}} \mathrm{k}_{\mathrm{LF}} \mathrm{C}_{\mathrm{eq}}^{1 / \mathrm{n}_{\mathrm{LF}}}}{1+\mathrm{k}_{\mathrm{LF}} \mathrm{C}_{\mathrm{eq}}^{1 / \mathrm{n}_{\mathrm{LF}}}}
\end{aligned}
$$

where $\mathrm{q}_{\mathrm{e}}\left(\mathrm{mg} \cdot \mathrm{g}^{-1}\right)$ is the adsorption capacity; $\mathrm{q}_{\mathrm{mL}}, \mathrm{q}_{\mathrm{mF}}$ and $\mathrm{q}_{\mathrm{mLF}}\left(\mathrm{mg} \cdot \mathrm{L}^{-1}\right)$ are the maximum adsorption capacities; $\mathrm{k}_{\mathrm{L}}, \mathrm{k}_{\mathrm{F}}$ and $\mathrm{k}_{\mathrm{LF}}\left(\mathrm{L}_{\mathrm{m}} \mathrm{mg}^{-1}\right)$ are the Langmuir, Freundlich and Langmuir-Freundlich adsorption constants, respectively; $\mathrm{n}_{\mathrm{F}}$ and $\mathrm{n}_{\mathrm{LF}}$ are the Freundlich and Langmuir-Freundlich adsorption intensity constants, respectively; and $\mathrm{C}_{\mathrm{eq}}\left(\mathrm{mg} . \mathrm{L}^{-1}\right)$ is the protein concentration at equilibrium. Parameter estimation was performed with help of the standard least squares method ${ }^{[21]}$ and was implemented in Mathematica 10.1 software, using the Nelder Mead method for minimization of the objective function ${ }^{[22]}$ and using a confidence level of 
$95 \%$. Statistical analyses and estimation of model parameters for the empirical statistical models were performed with the software STATISTICA 8.0 (StatSoft), with help of the standard least squares method, using a confidence level of $95 \%$. In order to facilitate the reading, obtained model representations and its correlation matrixes are presented in Supplementary Materials.

\section{Results and Discussions}

\subsection{Preliminary analyses}

Initially, preliminary correlation analyses (Table 2) were performed considering the variables (surfactant type, surfactant concentration and $\mathrm{O} / \mathrm{W}$ ratio) and the responses (size, zeta potential and BSA adsorption). As one can see, the type of surfactant was one of the analyzed design variables $\left(\mathrm{x}_{1}\right)$. As variable $\mathrm{x}_{1}$ has a discrete nature, replicates were performed at the central point of the continuous variables for both discrete variables (SDS and CTAB) and statistical analyses were conducted considering all experiments simultaneously and blocks generated by each discrete variable.

As one can observe in Table 2, BSA adsorption and zeta potential responses are correlated significantly with the surfactant type. This behavior can be explained in terms of the surfactant nature (anionic or cationic), since the ionic characteristics of the surfactant affects the surface charge of the particle and the adsorption process ${ }^{[23]}$. In addition,

Table 2. Correlation matrix for preliminary analyses.

\begin{tabular}{ccccccc}
\hline \multicolumn{7}{c}{ Correlation Matrix } \\
\hline & $\mathbf{x}_{\mathbf{1}}$ & $\mathbf{x}_{\mathbf{2}}$ & $\mathbf{x}_{\mathbf{3}}$ & Size & ZP & BSA \\
\hline $\mathrm{x}_{1}$ & 1.00 & 0.29 & 0.00 & 0.04 & 0.99 & -0.76 \\
$\mathrm{x}_{2}$ & 0.29 & 1.00 & 0.00 & -0.86 & 0.26 & -0.78 \\
$\mathrm{x}_{3}$ & 0.00 & 0.00 & 1.000 & 0.02 & -0.01 & -0.02 \\
Size & 0.03 & -0.86 & 0.02 & 1.00 & 0.08 & -0.61 \\
ZP & 0.99 & 0.26 & 0.01 & 0.08 & 1.00 & -0.73 \\
BSA & -0.76 & -0.79 & -0.02 & -0.61 & -0.73 & 1.00 \\
\hline
\end{tabular}

BSA adsorption is also significantly correlated with surfactant concentration, particle size and zeta potential responses. This indicates that the amount of surfactant can significantly affect the protein adsorption process and the functionalization of the obtained polymer particles. Such behaviour has been usually overlooked in most publications in the field, which tend to concentrate on the reaction mechanism of functionalization. As a matter of fact, protein adsorption onto polymer NPs can constitute a complex process, depending on the size and surface charge of the particles, rendering the role of the surfactant very important ${ }^{[15]}$. Regarding the average particle sizes, it presented strong negative correlation with the surfactant concentration, as it might already be expected ${ }^{[24]}$.

Table 3 presents the experimental responses for average sizes, PdI and zeta potential for the miniemulsion droplets and polymer NPs. Conversion and BSA adsorption results are also presented in Table 3. Most tests achieved maximum conversions after 2 hours of reaction, as one might expect given the small average particle sizes and the high reactivity of the MMA monomer ${ }^{[25]}$. However, reactions performed with lower organic loads $(15 \%$ of $\mathrm{O} / \mathrm{W})$ and higher surfactant concentrations (5 wt\%) (Tests 3 and 7) exhibited reduced conversions for both surfactants, indicating that the use of very high amounts of surfactant could negatively affect the evolution of the miniemulsion reaction, an aspect that has not been discussed in the literature.

Table 3 presents the experimental responses for average sizes, PdI and zeta potential for the miniemulsion droplets and polymer NPs. Conversion and BSA adsorption results are also presented in Table 3. Most tests achieved maximum conversions after 2 hours of reaction, as one might expect given the small average particle sizes and the high reactivity of the MMA monomer ${ }^{[25]}$. However, reactions performed with lower organic loads ( $15 \%$ of $\mathrm{O} / \mathrm{W})$ and higher surfactant concentrations ( $5 \mathrm{wt} \%$ ) (Tests 3 and 7 ) exhibited reduced conversions for both surfactants, indicating that the use of very high amounts of surfactant could negatively affect the evolution of the miniemulsion reaction, an aspect that has not been discussed in the literature.

Table 3. Experimental responses.

\begin{tabular}{|c|c|c|c|c|c|c|c|c|c|c|c|}
\hline \multirow[t]{2}{*}{ Run } & \multirow[t]{2}{*}{ Surfactant } & \multirow{2}{*}{$\begin{array}{c}\text { Surfactant } \\
\text { Concentration }\end{array}$} & \multirow{2}{*}{$\begin{array}{c}\text { O/W phase } \\
\text { ratio }\end{array}$} & \multirow{2}{*}{$\begin{array}{c}\text { Conversion } \\
(\%)\end{array}$} & \multicolumn{2}{|c|}{ Size (nm) } & \multicolumn{2}{|c|}{ PdI } & \multicolumn{2}{|c|}{$\begin{array}{c}\text { Zeta Potential } \\
(\mathrm{mV})\end{array}$} & \multirow{2}{*}{$\begin{array}{c}\text { BSA } \\
\text { adsorption } \\
\left(\mathrm{mg} / \mathrm{m}^{2}\right)\end{array}$} \\
\hline & & & & & Droplet & Particle & Droplet & Particle & Droplet & Particle & \\
\hline 1 & -1 & -1 & -1 & 100 & 98.63 & 157.37 & 0.092 & 0.013 & -48.87 & -43.33 & 7.16 \\
\hline 2 & -1 & -1 & +1 & 100 & 158.03 & 149.07 & 0.316 & 0.023 & -48.53 & -49.63 & 6.66 \\
\hline 3 & -1 & +1 & -1 & 84.94 & 106.30 & 55.51 & 0.137 & 0.064 & -44.77 & -52.87 & 2.88 \\
\hline 4 & -1 & +1 & +1 & 100 & 125.97 & 64.48 & 0.207 & 0.068 & -34.20 & -43.70 & 3.44 \\
\hline 5 & +1 & -1 & -1 & 2.69 & 94.15 & $-{ }^{a}$ & 0.194 & $-{ }^{a}$ & +26.37 & $-{ }^{a}$ & $-{ }^{a}$ \\
\hline 6 & +1 & -1 & +1 & 2.68 & 89.59 & $-{ }^{a}$ & 0.284 & $-{ }^{a}$ & +13.07 & $-{ }^{a}$ & $-{ }^{a}$ \\
\hline 7 & +1 & +1 & -1 & 69.37 & 71.05 & 72.01 & 0.101 & 0.019 & +15.97 & +42.53 & 1.12 \\
\hline 8 & +1 & +1 & +1 & 99.88 & 81.73 & 75.66 & 0.355 & 0.012 & +35.60 & +34.60 & 0.71 \\
\hline 9 & -1 & 0 & 0 & 100 & 91.43 & 72.19 & 0.299 & 0.011 & -42.10 & -49.67 & 3.91 \\
\hline 10 & -1 & 0 & 0 & 100 & 75.43 & 71.39 & 0.183 & 0.033 & -55.07 & -54.27 & 3.77 \\
\hline 11 & -1 & 0 & 0 & 99.89 & 70.34 & 76.59 & 0.331 & 0.019 & -52.60 & -56.90 & 4.09 \\
\hline 12 & +1 & 0 & 0 & 99.55 & 115.87 & 109.77 & 0.108 & 0.195 & +25.73 & +44.13 & 1.74 \\
\hline 13 & +1 & 0 & 0 & 100 & 90.86 & 105.20 & 0.176 & 0.008 & +33.93 & +41.70 & 2.41 \\
\hline 14 & +1 & 0 & 0 & 99.66 & 98.03 & 111.57 & 0.160 & 0.152 & +37.67 & +40.17 & 2.13 \\
\hline
\end{tabular}

${ }^{\mathrm{a}}$ not measured. 
As exposed in Table 3, some reaction runs (using SDS as surfactant) showed a significant reduction of the average particle sizes, suggesting the occurrence of micellar nucleation, which can indicate the existence of significant mass transfer resistance of monomer from previously formed nanoparticles (containing hexadecane co-surfactant) to newly nucleated particles. Apparently, this very interesting reaction effect has been neglected in previous publications, as surfactant excess is avoided on purpose in miniemulsion polymerizations (see Supplementary Material). It is important to report that monomer conversion is normally expected to increase when the surfactant concentration is increased ${ }^{[26]}$, due to the increase of the number of polymer particles through micellar nucleation, loci of the polymerization reactions. However, classical models neglect the presence of co-stabilizers in the initially dispersed monomer droplets (usually prepared in absence of co-stabilizers in classical emulsion polymerizations), which prevent mass transfer of monomer from dispersed droplets to newly formed polymer particles.

In the case of $\mathrm{CTAB}$, the reduction of the organic load caused significant reduction of the zeta potential of droplets, indicating the preferential formation of micelles, which could lead to similar mass transfer limitations. Finally, tests 5 and 6 indicated the loss of emulsion stability, showing the lower coverage efficiency provided by CTAB and explaining the very low achieved monomer conversions.

According to Table 3, monomer droplets with average diameters in the nanometric region were obtained in all cases, validating the use of the sonicator for the initial droplet dispersion. Zeta potential results showed negative values for samples prepared with SDS and positive values for samples prepared with CTAB. This result might already be expected, given the ionic nature of each particular surfactant, as SDS is an anionic surfactant and CTAB is a cationic surfactant. In addition, the obtained values were higher than $30 \mathrm{mV}$ (in absolute values) in most experimental conditions, suggesting the high concentration of surfactants on the NPs surfaces and indicating that the dispersion stability was controlled by charge repulsion and charge distribution on the NPs surfaces. High dispersion stability is very important for actual applications, ensuring the preservation of the morphological properties of the final latex at rest for long periods of time.

Figure 1 shows the comparison between produced droplets and nanoparticles, for the two surfactants used, cationic and anionic. These results are consistent with the expected miniemulsion polymerization mechanism, as the initial monomer droplets are nucleated by free radicals and behave as 'nanoreactors', keeping the original size and interfacial charge essentially constant throughout the reaction course ${ }^{[27]}$. However, when extended to the other analyzed experimental conditions, the obtained results were somewhat different, as shown in Figure 1. Particle sizes (Figure 1-A) were equivalent to the droplet sizes when CTAB was used as surfactant, confirming the stability of the emulsion. However, when SDS was used as surfactant, growth of particle sizes was observed when the surfactant
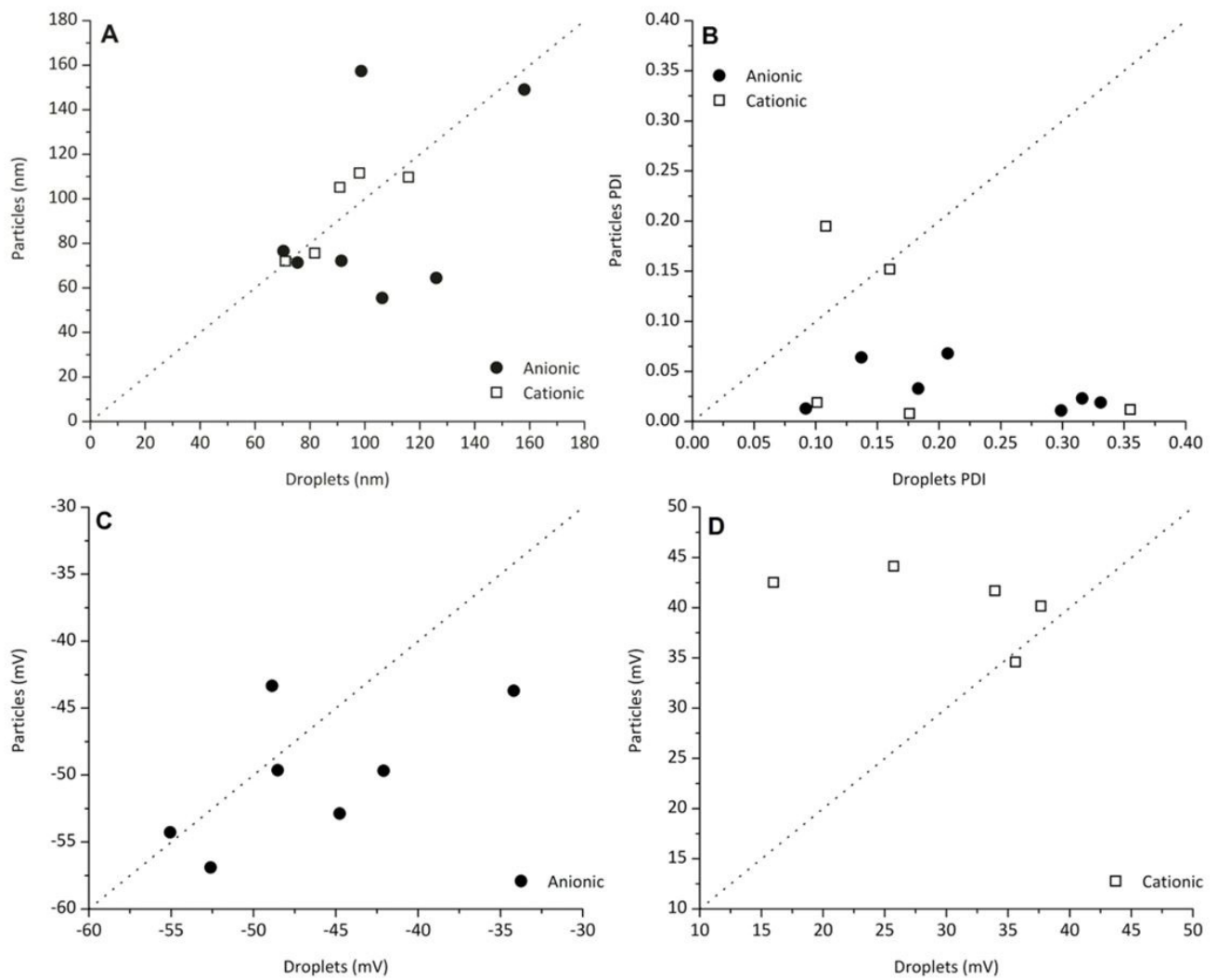

Figure 1. Comparison between produced droplets and nanoparticles (cationic and anionic). (A) Size; (B) PdI; (C and D) Zeta potential. 
concentration was smaller (due to mild agglomeration) and reduction of particle sizes was observed when the SDS was larger (due to micellar nucleation). It is interesting to observe that the reactor operation seemed to be more sensitive to modification of the SDS concentrations than to modification of the $\mathrm{CTAB}$ concentration, making the use of CTAB more advantageous in respect to control of the reaction conditions. In general, it is important to emphasize that size distributions of the droplets were more dispersed (Figure 1-B) (higher PdI) than the polymer NPs (Figure S1 - Supplementary Material), indicating the occurrence of mass transfer effects during the reaction, possibly because of the large range of analyzed surfactant concentrations ${ }^{[24]}$.

Regarding the zeta potential (Figure 1-C and Figure 1-D), polymer particles usually presented higher electrical charges (in absolute values) than the droplets. As this charge concentration effect was very significant in some cases, this cannot be assigned only to volume reduction caused by the higher density of the polymer material. This effect probably indicates that the particle-surfactant interaction changed during the course of the reaction, due to the continuous modification of the properties of the particle surface and of the solution. This complex dynamic feature of the particle-surfactant interaction, which certainly depends on the chemical, thermodynamic and electronic characteristics of the involved chemical species, has yet to be analyzed in detail in the literature, as it has been implicitly assumed that the charge distribution remains constant throughout the reaction course.

Regarding the BSA adsorption, polymer particles produced with SDS showed higher adsorption efficiencies than particles produced with $\mathrm{CTAB}$ at the analyzed conditions, confirming that the amount of adsorbed protein is controlled mainly by the surface charge. This behaviour makes the initial emulsion formulation fundamental for proper understanding of the final protein functionalization of the particle, an issue that has been frequently overlooked in the open literature, which tends to concentrate the analyses on the chemical characteristics of the produced polymer materials and to neglect the relevant role of the surfactant on the performance of the functionalization step.

\subsection{Statistical analyses for polymer NPS}

The statistical models used to describe average sizes, zeta potential and BSA adsorption as functions of the process parameters had the general form (Equation 4):

$$
y^{c}=a_{0}+\sum_{i=1}^{N X} a_{i} x_{i}+\sum_{i=1}^{N X} \cdot \sum_{j=i+1}^{N X} b_{i j} x_{i} x_{j}+c x_{i}^{2}
$$

where $\mathrm{y}^{\mathrm{c}}$ is the model response, $\mathrm{a}_{0}$ is the response bias, $\mathrm{a}_{\mathrm{i}}$ are the main variable effects, $b_{i j}$ are the synergetic interaction effects and $c$ is a nonlinear quadratic effect. Statistical models were built iteratively. Initially, all analyzed effects were assumed to exist. Then, insignificant (within the 95\% confidence level) parameters were discarded and the model was rebuilt. The procedure was repeated until attaining a full set of statistically significant parameters. Then, the model quality was evaluated, using the minimum value of the objective function and the experimental variance at the central point to perform the Chi-square test, with confidence level of $95 \%$. Besides, the experimental variance and the model prediction variance were compared with the F-test, with confidence level of $95 \%$. Models were accepted only when all these conditions were satisfied.

One must observe that variable $\mathrm{x}_{1}$ is discrete, meaning that $\mathrm{x}_{1}$ can only assume the values $(-1)$ or $(+1)$. Therefore, the coefficients that multiply the variable $x_{1}$ indicate the effect of changing the type of surfactant in the particularly analyzed statistical effect. As a consequence, one may assume that Equation (4) summarizes two distinct equations: one for SDS (when $\mathrm{x}_{1}$ is made equal to -1 ) and one for CTAB (when $\mathrm{x}_{1}$ is made equal to +1 ). The simultaneous analyses of all available experimental data is advantageous because leads to higher number of degrees of freedom and more precise estimation of the model parameters.

Regarding the average sizes of the PMMANPs, the obtained model (Equation 5) showed that surfactant concentration was the most significant variable $\left(a_{2}\right)$, exerting a negative effect on the average particle size, indicating that the increase of the surfactant concentration caused the reduction of average particle sizes, as well documented in the literature ${ }^{[28,29]}$. It is important to observe that the surfactant type also exerted a significant effect on average particle sizes, as the cationic surfactant shifted the average sizes towards higher values $\left(a_{1}\right)^{[30]}$. Despite that, the use of CTAB made the system even more sensitive to modification of the surfactant concentration $\left(b_{12}\right)$, indicating the less efficient stabilization of the NPs promoted by CTAB, despite the apparent higher sensitivity of particle diameters to change of the SDS concentrations, as described in the previous section. Finally, it must be observed the negligible effect exerted by the organic load on the average particle sizes $\left(b_{23}\right)$ and the existence of quadratic nonlinear effects $(c)$ associated with the modification of the process parameters. Although the proposed experimental design does not allow for unambiguous identification of the quadratic effect, it was assumed that this effect was related to the surfactant concentration, given its much larger influence on the analyzed process response.

$$
\begin{aligned}
& \text { Size }=(91 \pm 3)+(18 \pm 3) \cdot x_{1}-(57 \pm 4) \cdot x_{2}- \\
& (11 \pm 4) \cdot x_{1} \cdot x_{2}+(4 \pm 3) \cdot x_{2} \cdot x_{3}+(33 \pm 5) \cdot x_{2}^{2}
\end{aligned}
$$

The analysis of the zeta potential showed that this process response was influenced mainly by the type of surfactant $\left(a_{1}\right)$, as shown in Equation 6. The surfactant molecules are located on the surfaces of the particles and define the observed charge. As expected, particles produced with SDS were negative, while the ones prepared with $\mathrm{CTAB}$ showed a positive zeta potentia ${ }^{[31]}$. It is important to observe the lower absolute surface charge provided by CTAB, which can possibly be related to its lower stabilizing effect, when compared to SDS.

$$
\text { Zeta Potential }=-(5 \pm 3)+(45 \pm 3) \cdot x_{1}
$$

A statistical model was also used to evaluate the influence of the process variables on the BSA adsorption onto PMMA NPs. Equation 6 shows that both variables, surfactant type and concentration, exerted significant effects on the adsorption process. This can be regarded as a very important result, since the role of surfactant on bioconjugation processes has 
been largely neglected, as discussed previously. Adsorption assays were performed in acetate buffer $\mathrm{pH}=4.5$, where BSA presents null charge ${ }^{[15,32]}$. This condition was selected on purpose, considering that the protein presents better adsorptive properties in the isoelectric point and the obtained results should not be influenced by the protein charge ${ }^{[33]}$. According to Equation 7, the surfactant concentration was once more the most influential process parameter $\left(a_{2}\right)$. Besides, the cationic surfactant shifted protein adsorption towards lower values $\left(a_{1}\right)$, a result that can possibly be related to its lower stabilizing capacity (and lower absolute surface charges). It should be noted the occurrence of less important nonlinear effects $\left(b_{23}\right.$ and $\left.c\right)$.

$$
\begin{aligned}
& \mathrm{BSA}=(2.9 \pm 0.2)-(1.0 \pm 0.2) \cdot \mathrm{x}_{1}-(1.9 \pm 0.2) \cdot \mathrm{x}_{2}+ \\
& (0.3 \pm 0.2) \cdot \mathrm{x}_{2} \cdot \mathrm{x}_{3}+(1.1 \pm 0.2) \cdot \mathrm{x}_{2}^{2}
\end{aligned}
$$

BSA adsorption using CTAB were smaller than when SDS was used, indicating that the amount of adsorbed protein can be controlled by the surface charge of the polymer particles. These results show that protein adsorption is significantly influenced by the electrostatic interaction between the protein and the adsorbent, as reported by Patil et al. ${ }^{[23]}$, as the surface charge can modify the electrostatic interaction between nanoparticles and the proteins. Nandhakumar et al. ${ }^{[34]}$ found that poly( $\varepsilon$-caprolactone) nanoparticles positively charged by CTAB showed higher adsorption efficiency of HSA (human serum albumin) than negatively charged particles by SDS. However, these authors performed the experiments above the isoelectric point of the protein, making it negatively charged, leading to larger affinity with the positively charged nanoparticles. Thus, an important factor to be taken into account during protein bioconjugation through adsorption is the protein charge (which is influenced by its isoelectric point and by the $\mathrm{pH}$ of the medium) and the polymer NP charge, affected mostly by the surfactant.

\subsection{Statistical analyses for polymer NPs for each surfactant}

The discussion presented above showed that the type of surfactant exerted a dominant and pronounced effect on the analyzed properties, which can eventually hide and confound the less important effects exerted by the remaining variables. For this reason, analyses of variable effects on average sizes, zeta potential and BSA adsorption data were performed for each analyzed surfactant independently, following the very same procedures described previously. As one can see in Equation 8 and Equation 9, built with SDS data, surfactant concentration (parameter $a_{2}$ ) was the variable that exerted the highest influence on average particle sizes and BSA adsorption. Once again, the existence of significant nonlinear effects (parameters $b_{23}$ and $c$ ) could be observed. These results are consistent with the previously described models, as presented in Equations 5 and 7. The correlation among parameters (Supplementary Material) were equal to zero, with exception of the quadratic parameters, as expected. However, it was not possible to obtain a significant statistical model for zeta potential, which means that the type of surfactant controls this property, which is not affected by the remaining variables. This means that the particle surfaces were saturated with SDS in all analyzed conditions, showing that SDS molecules are preferentially adsorbed on the particle-water interfaces, regardless the particularly analyzed SDS concentrations and monomer loads.

$$
\begin{aligned}
& \text { Size }_{\text {SDS }}=(74 \pm 3)-(47 \pm 2) \cdot \mathrm{x}_{2}+ \\
& (4 \pm 2) \cdot \mathrm{x}_{2} \cdot \mathrm{x}_{3}+(33 \pm 4) \cdot \mathrm{x}_{2}^{2} \\
& \mathrm{BSA}_{\mathrm{SDS}}=(3.9 \pm 0.1)-(1.9 \pm 0.1) \cdot \mathrm{x}_{2}+ \\
& (0.26 \pm 0.1) \cdot \mathrm{x}_{2} \cdot \mathrm{x}_{3}+(1.1 \pm 0.2) \cdot \mathrm{x}_{2}^{2}
\end{aligned}
$$

Regarding $\mathrm{CTAB}$ data, Equations 10 and 11 represent the adjusted models for average particle sizes and BSA adsorption. As expected, the surfactant concentration was once more the most influential parameter $\left(a_{2}\right)$ to explain the average sizes and BSA adsorption ${ }^{[34]}$. For particle sizes, this result can explain the reaction behaviour in tests 5 and 6 , when massive agglomeration of polymer particles took place. Once again, it was not possible to obtain a significant statistical model for zeta potential, which means that the type of surfactant controls this property, which is not affected by the remaining variables. As already described, this means that the particle surfaces were saturated with CTAB in all analyzed conditions, showing that $\mathrm{CTAB}$ molecules are preferentially adsorbed on the particle-water interfaces, regardless the particularly analyzed CTAB concentrations and monomer loads.

$$
\begin{aligned}
& \text { Size }_{\text {CTAB }}=(109 \pm 4)-(35 \pm 6) \cdot \mathrm{x}_{2} \\
& \text { BSA }_{\text {CTAB }}=(2 \pm 4)-(1.2 \pm 0.6) \cdot \mathrm{x}_{2}
\end{aligned}
$$

\subsection{Adsorption analyses}

The zeta potential of the BSA-modified polymer NPs was evaluated after the adsorption process, as shown in Figure 2. Particles produced with SDS (initially charged negatively) presented positive charges, while particles produced with CTAB (initially charged positively) presented lower positive zeta potentials after adsorption. These results clearly indicate the modification of the PMMA NPs surfaces after adsorption of BSA.

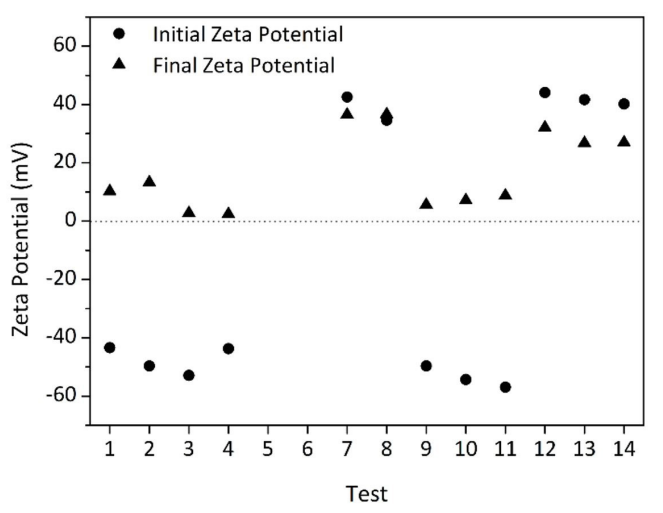

Figure 2. Zeta potentials of PMMA NPs before and after the BSA adsorption process. 

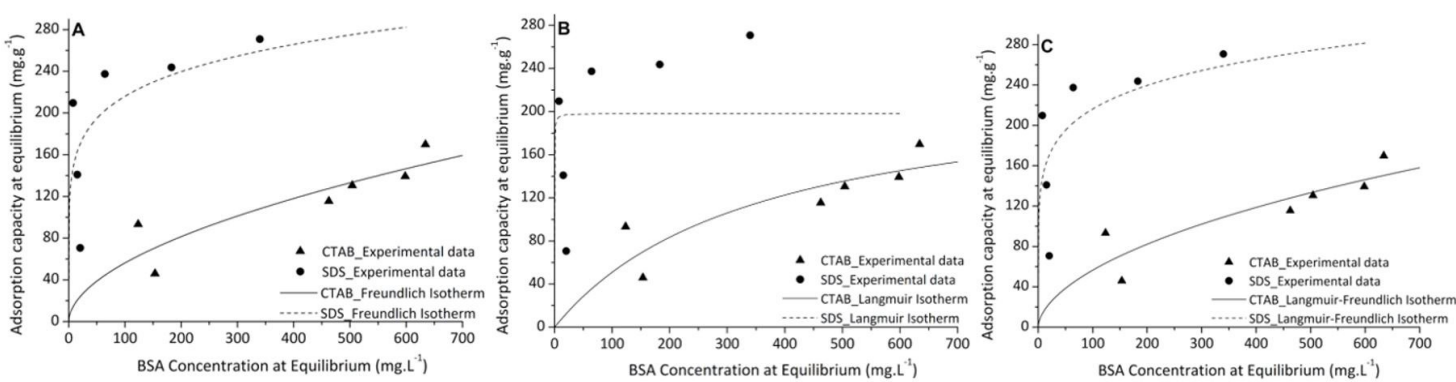

Figure 3. Fits of BSA adsorption isotherms for nanoparticles produced with SDS and CTAB. (A) Freundlich isotherm; (B) Langmuir isotherm; and (C) Langmuir-Freundlich isotherm.

Table 4. Estimated parameters for the analyzed adsorption models.

\begin{tabular}{cccc}
\hline Isotherm & Constants & SDS & CTAB \\
\hline Langmuir & $R^{2}$ & 0.8871 & 0.9725 \\
& $q_{M L}$ & 198.101 & 230.633 \\
& $k_{L}$ & 8.1991 & 0.0028 \\
Freundlich & $R^{2}$ & 0.9292 & 0.9766 \\
& $q_{M F}$ & 2.7202 & 1.8826 \\
& $k_{F}$ & 39.9292 & 2.5420 \\
Langmuir- & $n_{F}$ & 6.7046 & 1.8692 \\
Freundlich & $R^{2}$ & 0.9290 & 0.9762 \\
& $q_{M L F}$ & 6018.64 & 1259.43 \\
& $k_{L F}$ & 0.0184 & 0.0033 \\
& $n_{L F}$ & 6.5118 & 1.7418 \\
\hline
\end{tabular}

In order to better understand the BSA adsorption behaviour on the surface of the synthesized PMMA NPs, adsorption isotherms were built for PMMA NPs obtained at the central point of the proposed factorial design. Modeling the adsorption isotherms can be important when it is desired to compare the relative adsorption performances of different $\mathrm{NPs}^{[35]}$.

As shown in Figure 3, the surface charge represents a significant variable for analysis of BSA adsorption in PMMA NPs. Although both nanoparticles present favorable adsorption (concave profiles) ${ }^{[36]}$, nanoparticles prepared with SDS show higher adsorption capacity, as also described in Equation 7. All analyzed isotherm models provided satisfactory fits for the available data, with exception of the Langmuir model when applied to NPs prepared with SDS. Table 4 shows the estimated parameters for all studied adsorption models. It can be observed that Freundlich isotherm provided the best fits for both nanoparticles, suggesting a multilayer adsorption process ${ }^{[37]}$. This can be regarded as an important feature of protein immobilization onto nanoparticle surfaces, as the formation of protein monolayers has been frequently assumed in the open literature for interpretation of experimental data ${ }^{[38]}$.

\section{Conclusions}

PMMA NPs were produced through miniemulsion polymerizations at different reaction conditions, in order to evaluate the influence of the anionic surfactant SDS and the cationic $\mathrm{CTAB}$ on protein immobilization through adsorption. Obtained results showed that both surfactant type and surfactant concentration are the most significant variables to explain not only the size of the nanoparticles, but also the rate and efficiency of protein adsorption, exerting fundamental influence on functionalization of PMMA nanoparticles with proteins.

Particularly, PMMA NPs presented average sizes ranging from 55 to $157 \mathrm{~nm}$, with narrower polydispersities than the initial monomer droplets. The produced NPs presented high values of zeta potential, ranging from -57 to $+44 \mathrm{mV}$, controlled strictly by the type of surfactant, indicating that the particle-water interfaces were fully saturated with surfactant in all analyzed conditions and also indicating the preferential adsorption of both surfactants on the interfacial surfaces of the dispersed media. Regarding the BSA adsorption, the characteristics of the surfactant also controlled the adsorption efficiency, with enhanced rates of adsorption on the anionic particle surfaces, showing that the surfactant exerts fundamental effect on functionalization of emulsified polymer particles, which must be explicitly acknowledged in studies of polymer particle functionalization with proteins. Finally, BSA adsorption on PMMANPs was shown to follow a multilayer adsorption process, given the better fitting of available data obtained with the Freundlich model.

\section{Acknowledgments}

The authors thank CNPq (Conselho Nacional de Desenvolvimento Científico e Tecnológico, Brazil) for providing scholarships.

\section{References}

1. Hans, M. L., \& Lowman, A. M. (2002). Biodegradable nanoparticles for drug delivery and targeting. Current Opinion in Solid State and Materials Science, 6(4), 319-327. http:// dx.doi.org/10.1016/S1359-0286(02)00117-1.

2. El-Say, K. M., \& El-Sawy, H. S. (2017). Polymeric nanoparticles: promising platform for drug delivery. International Journal of Pharmaceutics, 528(1-2), 675-691. http://dx.doi.org/10.1016/j. ijpharm.2017.06.052. PMid:28629982.

3. Reis, C. P., Neufeld, R. J., Ribeiro, A. J., \& Veiga, F. (2006). Nanoencapsulation I. Methods for preparation of drug-loaded polymeric nanoparticles. Nanomedicine; Nanotechnology, Biology, and Medicine, 2(1), 8-21. http://dx.doi.org/10.1016/j. nano.2005.12.003. PMid:17292111. 
4. Nagavarma, B. V. N., Yadav, H. K. S., Ayaz, A., Vasudha, L. S., \& Shivakumar, H. G. (2012). Different techniques for preparation of polymeric nanoparticles- A review. Asian Journal of Pharmaceutical and Clinical Research, 5(3), 16-23.

5. Rao, J. P., \& Geckeler, K. E. (2011). Polymer nanoparticles: preparation techniques and size-control parameters. Progress in Polymer Science (Oxford), 36(7), 887-913. http://dx.doi. org/10.1016/j.progpolymsci.2011.01.001.

6. Elsabahy, M., \& Wooley, K. L. (2012). Design of polymeric nanoparticles for biomedical delivery applications. Chemical Society Reviews, 41(7), 2545-2561. http://dx.doi.org/10.1039/ c2cs15327k. PMid:22334259.

7. Zhenqian, Z., Sihler, S., \& Ziener, U. (2017). Alizarin Yellow R (AYR) as compatible stabilizer for miniemulsion polymerization. Journal of Colloid and Interface Science, 507(1), 337-343. http://dx.doi.org/10.1016/j.jcis.2017.08.007. PMid:28803027.

8. Wachsmann, P., Moulari, B., Béduneau, A., Pellequer, Y., \& Lamprecht, A. (2013). Surfactant-dependence of nanoparticle treatment in murine experimental colitis. Journal of Controlled Release, 172(1), 62-68. http://dx.doi.org/10.1016/j. jconrel.2013.07.031. PMid:23933520.

9. Lorca, B. S. S., Bessa, E. S., Nele, M., Santos, E. P., \& Pinto, J. C. (2012). Preparation of PMMA nanoparticles loaded with benzophenone-3 through miniemulsion polymerization. Macromolecular Symposia, 319(1), 246-250. http://dx.doi. org/10.1002/masy.201100252.

10. Fonseca, L. B., Nele, M., Volpato, N. M., Seiceira, R. C., \& Pinto, J. C. (2013). Production of PMMA nanoparticles loaded with praziquantel through "in situ" miniemulsion polymerization. Macromolecular Reaction Engineering, 7(1), 54-63. http://dx.doi.org/10.1002/mren.201200036.

11. Moreira, T. S., Oliveira, M. A. M., Lima, L. M. T. R., Souza, M. N., \& Pinto, J. C. C. S. (2014). Synthesis of nanoparticles loaded with tamoxifen by in situ miniemulsion RAFT polymerization. Macromolecular Symposia, 344(1), 101-107. http://dx.doi.org/10.1002/masy.201400025.

12. Schork, F. J., Luo, Y., Smulders, W., Russum, J. P., Butté, A., \& Fontenot, K. (2005). Miniemulsion polymerization. In M. Okubo (Ed.), Polymer particles (pp. 129-255). Berlin: Springer. http://dx.doi.org/10.1007/b100115.

13. Johnsson, B., Löfås, S., \& Lindquist, G. (1991). Immobilization of proteins to a carboxymethyldextran-modified gold surface for biospecific interaction analysis in surface plasmon resonance sensors. Analytical Biochemistry, 198(2), 268-277. http://dx.doi. org/10.1016/0003-2697(91)90424-R. PMid:1724720.

14. Carlson, R. (1992). Two-level factorial designs. In R. Carlson (Ed.), Design and optimization in organic synthesis (pp. 89122). New York: Elsevier Science

15. Yasuda, M., \& Ono, K. (2015). BSA adsorption and immobilization onto charged monodisperse polymer nanoparticles. Journal of Biosensors \& Bioelectronics, 6(4), 4-11. http://dx.doi. org/10.4172/2155-6210.1000183.

16. Bradford, M. M. (1976). A rapid and sensitive method for the quantitation of microgram quantities of protein utilizing the principle of protein-dye binding. Analytical Biochemistry, 72(12), 248-254. http://dx.doi.org/10.1016/0003-2697(76)90527-3. PMid:942051.

17. Vauthier, C., Schmidt, C., \& Couvreur, P. (1999). Measurement of the density of polymeric nanoparticulate drug carriers by isopycnic centrifugation. Journal of Nanoparticle Research, 1(3), 411-418. http://dx.doi.org/10.1023/A:1010031605547.
18. Langmuir, I. (1918). The adsorption of gases on plane surfaces of glass, mica and platinum. Journal of the American Chemical Society, 40(9), 1361-1403. http://dx.doi.org/10.1021/ja02242a004.

19. Freundlich, H. M. F. (1906). Over the adsorption in solution. Journal of Physical Chemistry, 57(1), 385-471.

20. Yoon, J.-Y., Park, H.-Y., Kim, J.-H., \& Kim, W.-S. (1996). Adsorption of BSA on highly carboxylated microspheres: quantitative effects of surface functional groups and interaction forces. Journal of Colloid and Interface Science, 177(2), 613620. http://dx.doi.org/10.1006/jcis.1996.0075.

21. Chen, S., Billings, S. A., \& Luo, W. (1989). Orthogonal least squares methods and their application to non-linear system identification. International Journal of Control, 50(5), 18731896. http://dx.doi.org/10.1080/00207178908953472.

22. Nelder, J. A., \& Mead, R. (1965). A simplex method for function minimization. The Computer Journal, 7(4), 308-313. http:// dx.doi.org/10.1093/comjn1/7.4.308.

23. Patil, S., Sandberg, A., Heckert, E., Self, W., \& Seal, S. (2007). Protein adsorption and cellular uptake of cerium oxide nanoparticles as a function of zeta potential. Biomaterials, 28(31), 46004607. http://dx.doi.org/10.1016/j.biomaterials.2007.07.029. PMid:17675227.

24. Hecht, L. L., Wagner, C., Landfester, K., \& Schuchmann, H. P. (2011). Surfactant concentration regime in miniemulsion polymerization for the formation of MMA nanodroplets by high-pressure homogenization. Langmuir, 27(6), 2279-2285. http://dx.doi.org/10.1021/la104480s. PMid:21314152.

25. Peixoto, A. C. B., Campos, I. M. F., Ferraz, H. C., \& Pinto, J. C. (2016). Use of Hydrophilic Monomers to Avoid Secondary Particle Nucleation in Miniemulsion Polymerizations of Methyl Methacrylate. Journal of Research Updates in Polymer Science, 5(2), 60-71. http://dx.doi.org/10.6000/1929-5995.2016.05.02.2.

26. Fontenot, K., \& Schork, F. J. (1993). Batch polymerization of methyl methacrylate in mini/macroemulsions. Journal of Applied Polymer Science, 49(4), 633-655. http://dx.doi. org/10.1002/app.1993.070490410.

27. Asua, J. M. (2002). Miniemulsion polymerization. Progress in Polymer Science, 27(7), 1283-1346. http://dx.doi.org/10.1016/ S0079-6700(02)00010-2.

28. Antonietti, M., \& Landfester, K. (2002). Polyreactions in miniemulsions. Progress in Polymer Science (Oxford), 27(4), 689-757. http://dx.doi.org/10.1016/S0079-6700(01)00051-X.

29. Pichot, R., Spyropoulos, F., \& Norton, I. T. (2010). O/W emulsions stabilised by both low molecular weight surfactants and colloidal particles: the effect of surfactant type and concentration. Journal of Colloid and Interface Science, 352(1), 128-135. http://dx.doi.org/10.1016/j.jcis.2010.08.021. PMid:20817195.

30. Qian, C., \& McClements, D. J. (2011). Formation of nanoemulsions stabilized by model food-grade emulsifiers using high-pressure homogenization: factors affecting particle size. Food Hydrocolloids, 25(5), 1000-1008. http://dx.doi. org/10.1016/j.foodhyd.2010.09.017.

31. Visaveliya, N., \& Köhler, J. M. (2014). Control of shape and size of polymer nanoparticles aggregates in a single-step microcontinuous flow process: a case of flower and spherical shapes. Langmuir, 30(41), 12180-12189. http://dx.doi. org/10.1021/la502896s. PMid:25251615.

32. Yoon, J. Y., Lee, J. H., Kim, J. H., \& Kim, W. S. (1998). Separation of serum proteins with uncoupled microsphere particles in a stirred cell. Colloids and Surfaces. B, Biointerfaces, 10(6), 365-377. http://dx.doi.org/10.1016/S0927-7765(97)00068-4. 
33. Gelamo, E. L., Silva, C. H. T. P., Imasato, H., \& Tabak, M. (2002). Interaction of bovine (BSA) and human (HSA) serum albumins with ionic surfactants: spectroscopy and modelling. Biochimica et Biophysica Acta, 1594(1), 84-99. http://dx.doi. org/10.1016/S0167-4838(01)00287-4. PMid:11825611.

34. Nandhakumar, S., Dhanaraju, M. D., Sundar, V. D., \& Heera, B. (2017). Influence of surface charge on the in vitro protein adsorption and cell cytotoxicity of paclitaxel loaded poly(e-caprolactone) nanoparticles. Bulletin of Faculty of Pharmacy, Cairo University, 55(2), 249-258. http://dx.doi.org/10.1016/j.bfopcu.2017.06.003.

35. Rabe, M., Verdes, D., \& Seeger, S. (2011). Understanding protein adsorption phenomena at solid surfaces. Advances in Colloid and Interface Science, 162(1-2), 87-106. http://dx.doi. org/10.1016/j.cis.2010.12.007. PMid:21295764.

36. Sun, C. J., Sun, L. Z., \& Sun, X. X. (2013). Graphical evaluation of the favorability of adsorption processes by using conditional langmuir constant. Industrial \& Engineering Chemistry Research, 52(39), 14251-14260. http://dx.doi.org/10.1021/ie401571p.

37. Foo, K. Y., \& Hameed, B. H. (2010). Insights into the modeling of adsorption isotherm systems. Chemical Engineering Journal, 156(1), 2-10. http://dx.doi.org/10.1016/j. cej.2009.09.013.

38. Dominguez-Medina, S., Blankenburg, J., Olson, J., Landes, C. F., \& Link, S. (2013). Adsorption of a protein monolayer via hydrophobic interactions prevents nanoparticle aggregation under harsh environmental conditions. ACS Sustainable Chemistry \& Engineering, 1(7), 833-842. http://dx.doi.org/10.1021/ sc400042h. PMid:23914342.

Received: Sept. 01, 2018

Revised: Mar. 24, 2019

Accepted: July 11, 2019 


\section{Supplementary Material}

Supplementary material accompanies this paper.

Legenda S.1 CMC Calculations

Legenda S.2 Comparisons between particle and droplet size distributions.

Legenda S.3 Statistical analyses

This material is available as part of the online article from http://www.scielo.br/po 